\title{
Finger Recognition by Feature Length
}

\author{
Ching-Liang Su \\ Department of Industrial Engineering \& Technology Management, Da Yeh University, \#168, University Road, Dacun, Chang-Hua, \\ 51505, Taiwan
}

\begin{abstract}
A small circle cake in a finger image is used to extract the partial finger image. Subsequently, the major axis length of this extracted circle cake is computed. Next, more circle cakes are extracted and the major axis length of each individual circle cake is computed. These obtained major axis lengths are chained together circularly to generate recognition features. These features are used to recognize a finger. The chaingenerated feature is compared to its adjacent features to locate the most match one. For every pixel in the sample image, the address of the match target in the unknown image is recorded. The trajectories from the sample image to unknown image can be similar and can be diverted significantly depending on the similarity of the two compared images. If the trajectories are similar, the match targets can hold together. Otherwise, the match targets are deviated to different places. The convergence and deviation situation can be used as an indictor to show whether the two compared images are coming from the identical source or not.
\end{abstract}

\section{Introduction}

This study uses a circle to extract the partial finger image. Subsequently, the major axis length of this extracted circle image is computed and obtained. Next, this major axis length is used as a feature to recognize a finger. The major axis length represents the feature of a circle image that a circle extracts. Instead of comparing a single pixel gray level value, now a circle region is compared. Therefore, the recognition rate is improved.

\section{Major axis length}

Figure 1(a) shows a pair of pliers. This pair of pliers has its own major axis. Figure 1(b) shows a staple case. This staple case has its own major axis as well. For any object

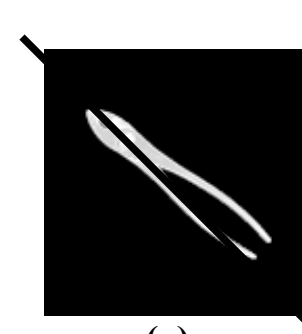

(a)

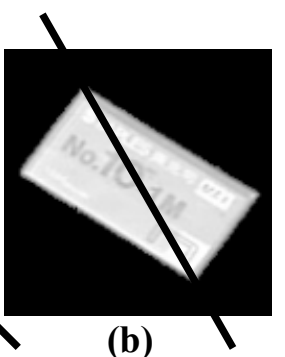

Fig. 1. Object major axis length. it has its own unique major axis. The length of the major axis can serve as a critical feature to identify the object. For the pair of pliers in figure 1(a), the pliers have its own unique major axis length. In figure 1(b) the staple case has its own unique major axis length. Figure 2(a), (b), and(c) show three pair of pliers. These pliers have three different poses. However, These pliers are from the identical source. The length of the major axis of each pair of pliers can be computed. As these pliers are from the same source, these three pliers have the same major axis length. The pliers in figure 2(a), (b), and (c) have different poses, they have the same unique major axis length. In the same way figure 3(a), (b), and(c) shows three different staple cases. These three staple cases are from the identical source. Although they have different poses, they have the same major axis length. This property is used to identify an object.

\footnotetext{
* Corresponding author: cls2@mail.dyu.edu.tw
} 


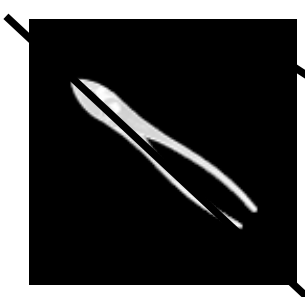

(a)

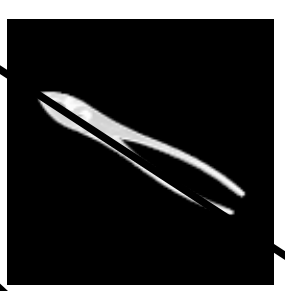

(b)

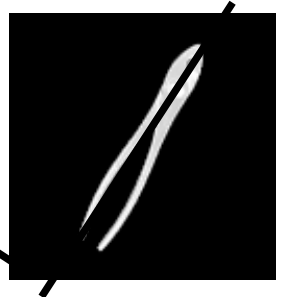

(c)

Fig. 2. Pliers with different orientations.

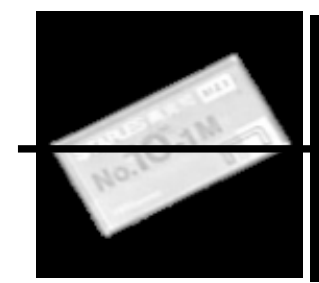

(a)

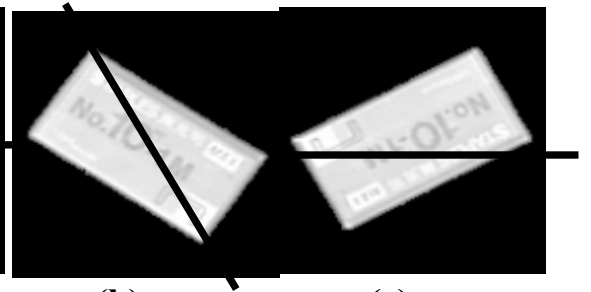

(b)

(c)

Fig. 3. Cases with different orientations.

\section{Computing thumb major axis}

Figure 4(a), (b), and (c) show three thumbs. Each thumb contains one circle. This circle is used to extract the partial thumb image. The major axis length of this partial thumb image is computed. Figure 4(a), figure 4(b), and figure 4 (c), individually each figure has its own extracting circle. Each extracting circle obtains its own circle image. Each extracting image has its own major axis length. The obtained major axis lengths are used to identify the different thumbs. Figure 4(a), (b), and (c) has one extracting circle. Figure 5(a) has one extracting circle too. However, in figure $5(\mathrm{~b})$, it has five extracting circles. These five circles are used to extract the thumb image. One can keep on adding extracting circle to figure 5(b). If more circles add to the thumb in figure 5(b), more major axis lengths can be obtained. Figure 6(a) contains one extracting circle. The centroid of this circle is $(\mathrm{x}, \mathrm{y})$. Correspondingly, one can locate the same corresponding position $(\mathrm{x}, \mathrm{y})$ in figure $6(\mathrm{~b})$. When the major axis length of the extracting circle in figure $6(\mathrm{a})$ is determined, this major axis length is saved in the $(\mathrm{x}, \mathrm{y})$ position in figure 6(b). Figure 6(b) shows the computed results of the major axis lengths. When every pixel in figure $6(\mathrm{a})$ is used as centroid to compute the major axis length, the result of figure $6(\mathrm{~b})$ can be obtain.

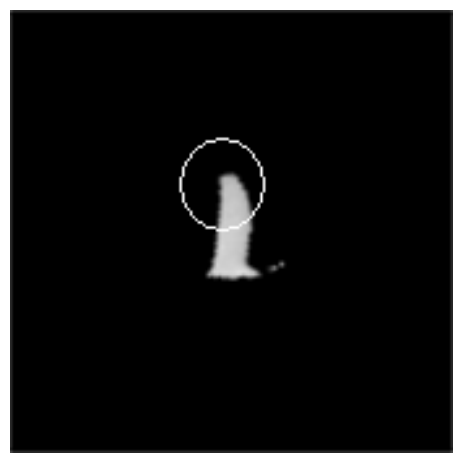

(a)

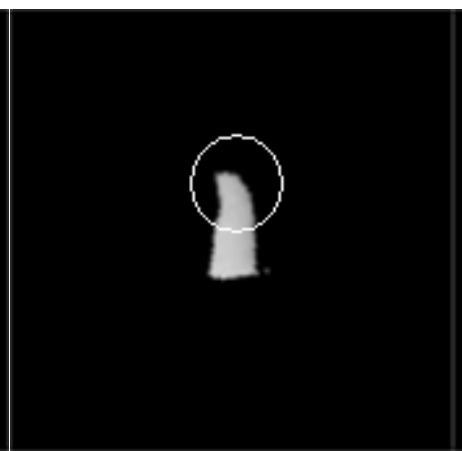

(b)

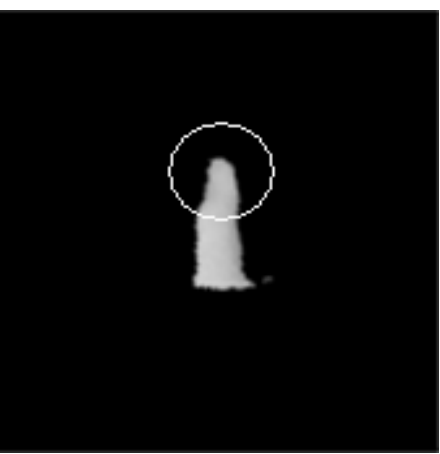

(c)

Fig. 4. Computing major axis lengths of that specific regions. 


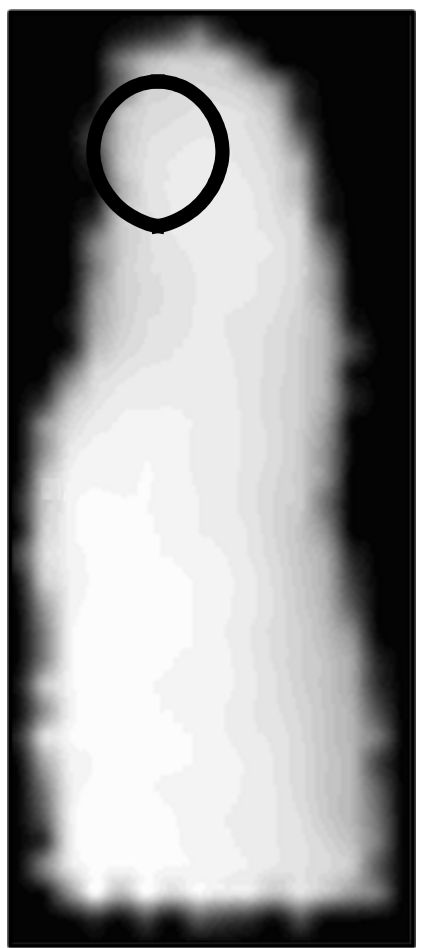

(a)

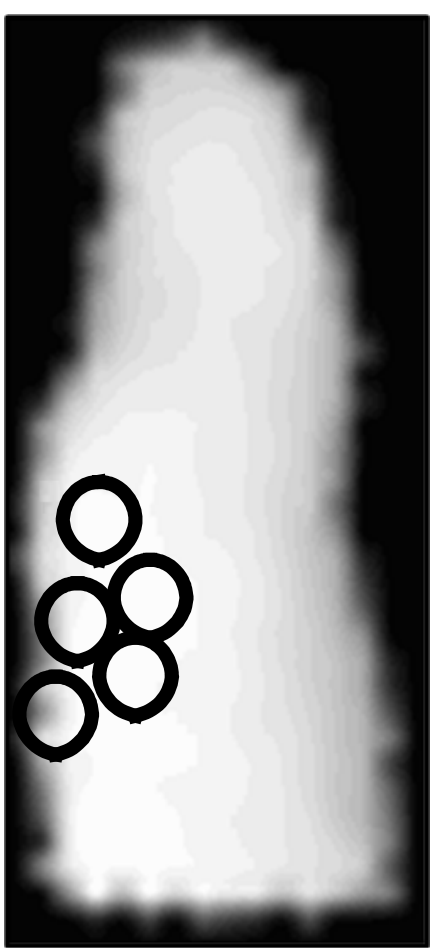

(b)

Fig. 5. Computing major axis lengths of that specific regions.

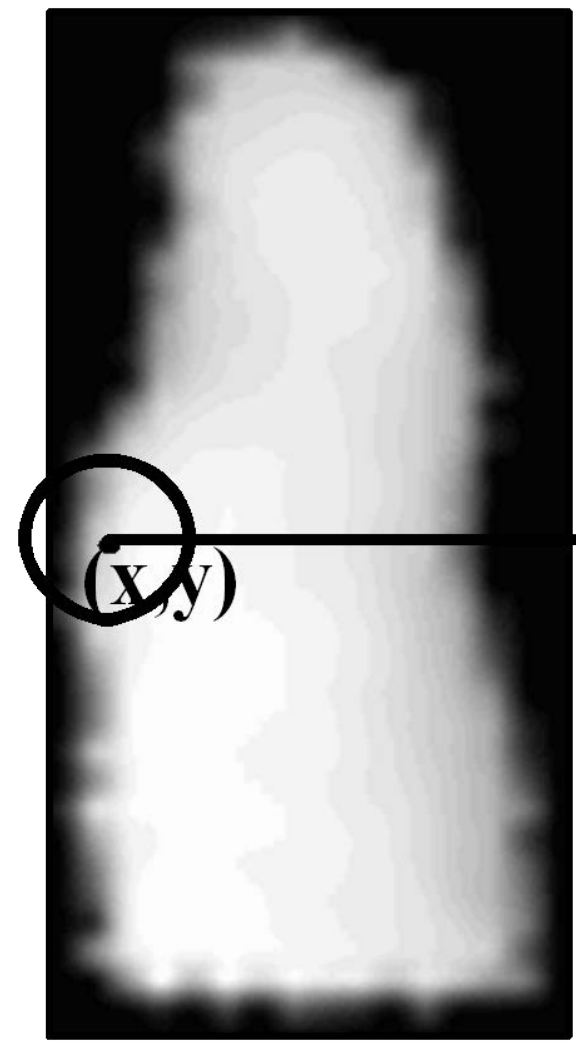

(a)

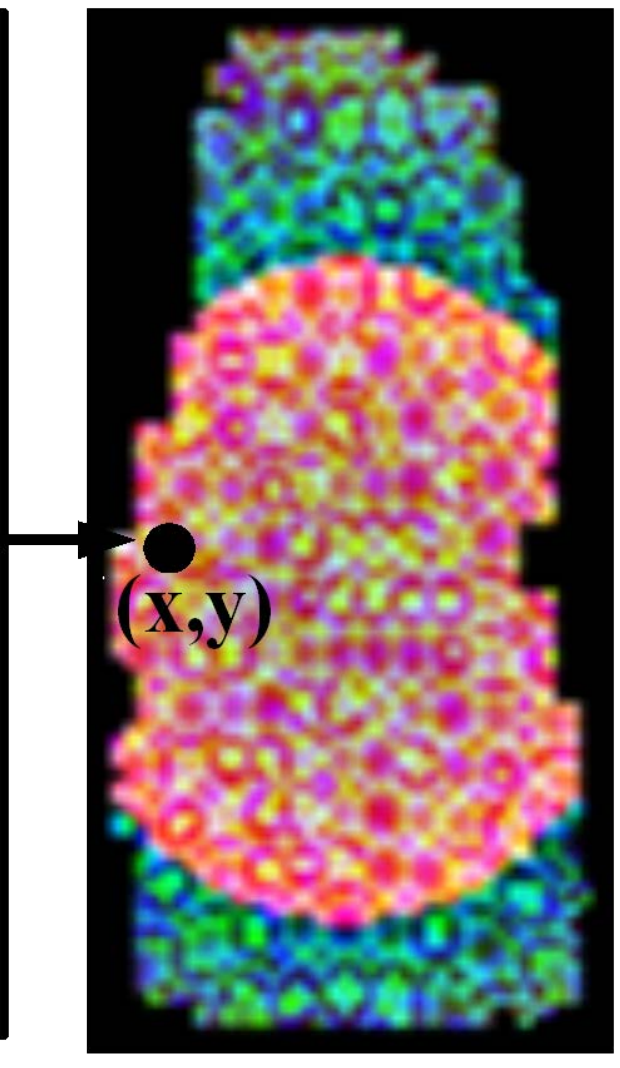

(b)

Fig. 6. Results after major axis length computations. 


\section{Converging or diverging comparison}

Figure 7(a) shows four black cakes. Each black cake represents a cake image. Furthermore, Each black cake has its own major axis length. For each cake, the major axis length is computed; they are: $22,8,3$, and 10, respectively. The finger can rotate to different directions. After the finger rotation, figure 7(a) can change to 7(b). If the finger is rotated further, figure 7 (c) can be obtained. However, although figures 7(a), (b), and (c) are rotated to different positions, summing the major axis length 22 , 8,3 , and 10 of the black cakes in figures $7(a),(b)$, and (c), $43 \mathrm{~s}$ can be obtained. Therefore, although a finger is rotated to different orientations, figure 7(a), (b), and (c) can generate the same value $43 \mathrm{~s}$. Thus, this method can solve the finger rotation problem. At the same time, this method can make the finger has a more salient feature. The pixel value is changed from the plain pixel value to major axis summing value. In this case, originally the pixel value of the centroid of figure 7(a) is photo-taken pixel value. Now it can change to the summing major axis values of eight different cakes, shown in the centroid of figure 7(a).

Figure 8 shows the palm, thumb, index, middle, ring, and small fingers. As the roots and fingertips cause errors, they are eliminated. The aforementioned procedures of figure 7 are performed to these fingers and the joined chain major axis lengths are obtained. The images after applied joined chain major-axis-length method are shown in the bottom row in figure 8 . These images are used to identify a person.

Figure 9(a) shows the centroid pixel, marked by X, that is pixel 0 . This is the sample finger. Figure $9(\mathrm{~b})$ is the unknown finger. Figure 9(b) shows eight square blocks, which represent pixels $0,1,2,3,4,5,6,7,8$. Pixel 0 in figure 9(a) is comparing to all the eight blocks in figure 9(b) to locate the most match pixel. The comparing method in this study is not simple one pixel to one pixel comparison. Instead, it searches the adjacent pixels to locate the most match one, as shown in figures 9(a) and (b). This method can increase the recognition rate. Figure 10(a) shows the sample image. Figure 10(b) shows the unknown image. In figure 10(a), the pixels 123456789 individually locate its most match pixel in figure 10(b). In this case, the trajectories of the pixels in figure 10(a) locate their most match pixels are similar. Pixels 123456789 in figure $10(\mathrm{~b})$ represent the most match target of pixels 123456789 in figure 10(a). In figure 10(a), the pixels 123456789 are held together. In figure 10(b), the pixels 123456789 are held together too. This shows the figures 10(a) and (b) are identical fingers. The situations of ABCDEFGHI and abcdefghi can explain the same situation that the sample and unknown images are coming from the same source. Figure 11(b) shows the trajectories are deviated to different places, as 123456789, ABCDEFGHI, and abcdefghi being displaced to different places. This shows the unknown figure 11(b) and sample figure 11(b) fingers are different fingers.

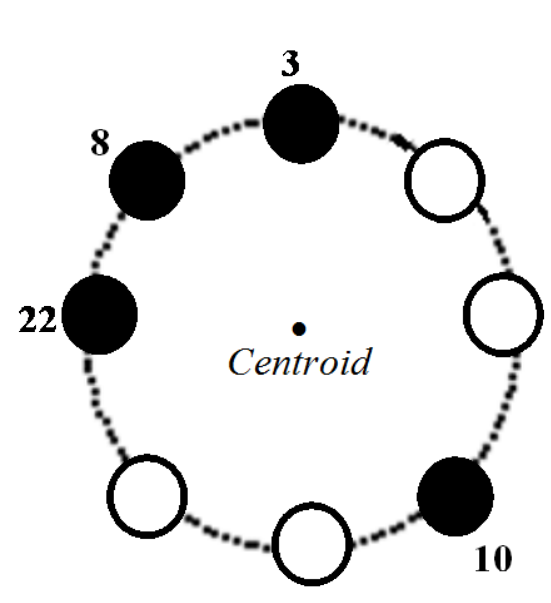

(a)

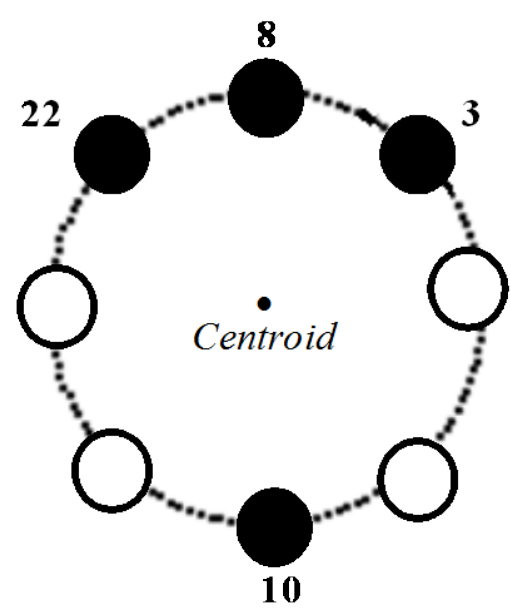

(b)

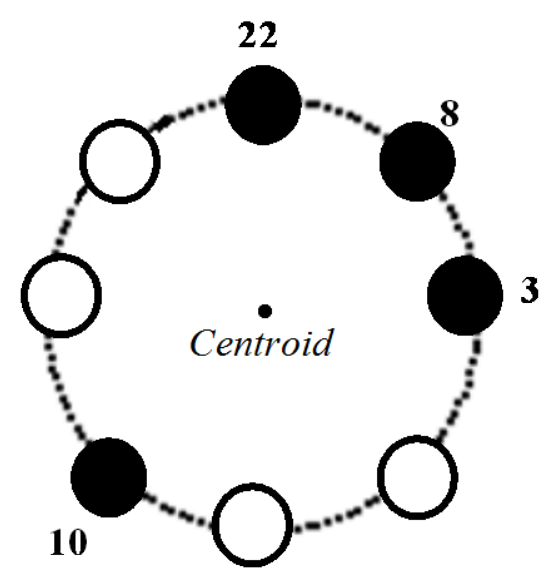

(c)

$22,8,3,10$, represent the length of major axis.

Fig. 7. The extracted major axis lengths, (a), (b), (c) are coming from the identical source. 

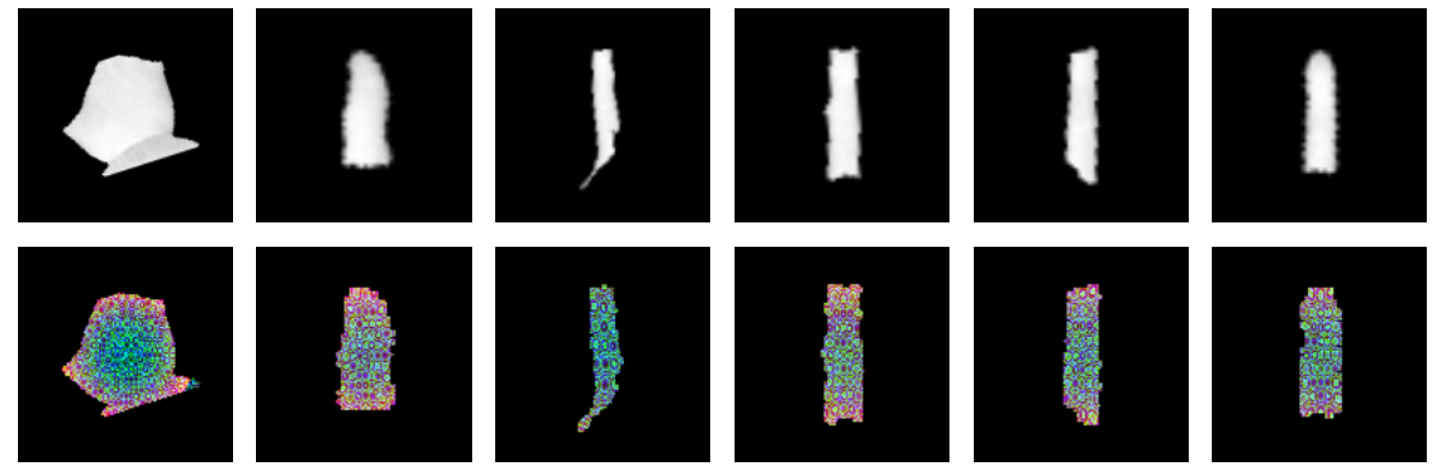

Fig. 8. Results after major axis length computations.

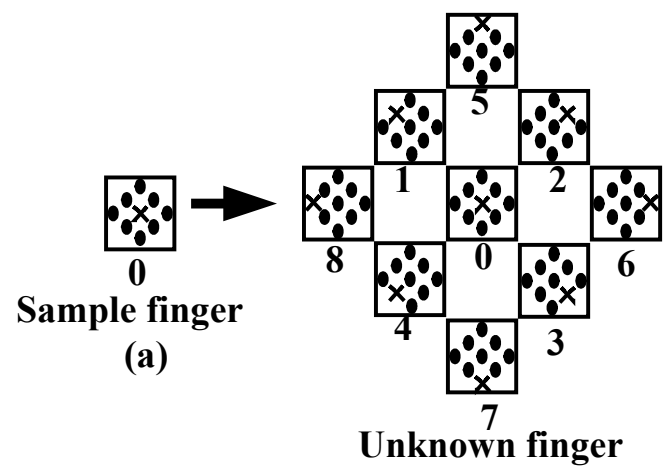

(b)

Fig. 9. Position 0 of sample finger comparing with positions $0,1,2, \ldots, 8$ of unknown finger.

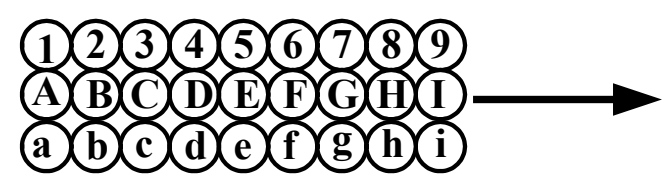

(a)Sample image

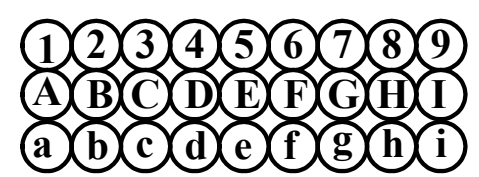

(b)Unknown image

Fig. 10. After comparisons the matching points are converged.

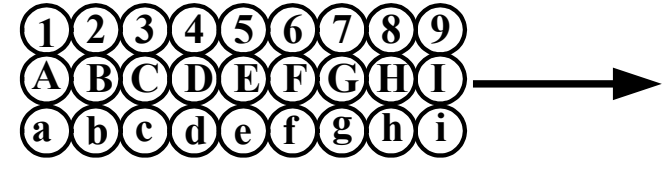

(a)Sample image

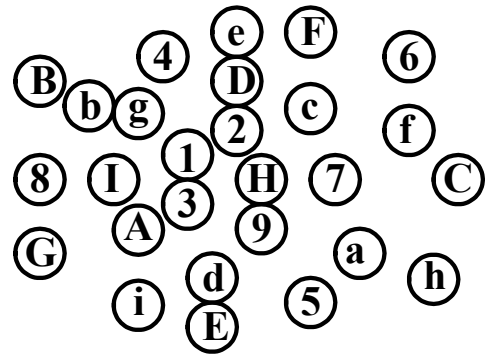

(b)Unknown image

Fig. 11. After comparisons the matching points are diverted.

\section{References}

1. Miguel A. Ferrer and Aythami Morales, "HandShape Biometrics Combining the Visible and ShortWave Infrared Bands," IEEE transactions on information forensics and security, vol. 6, no. 4, December 2011
2. Antonio Iula and Michele De Santis, "Experimental evaluation of an ultrasound technique for the biometric recognition of human hand anatomic elements," Ultrasonics 51 (2011) 683-688

3. Ching-Liang Su, "Ear Recognition by Major Axis and Complex Vector Manipulation," KSII TRANSACTIONS ON INTERNET AND INFORMATION SYSTEMS, 11 (2017) 1650-166 